\title{
Sources of Electrical Brain Activity Most Relevant to Performance of Brain-Computer Interface Based on
}

\section{Motor Imagery}

\author{
Alexander Frolov, Dušan Húsek, Pavel Bobrov, \\ Olesya Mokienko and Jaroslav Tintera
}

Additional information is available at the end of the chapter

http://dx.doi.org/10.5772/55166

\section{Introduction}

A brain-computer interface $(\mathrm{BCI})$ provides a direct functional interaction between the human brain and the external device. Many kinds of signals (from electromagnetic to metabolic $[23,38,42])$ could be used in BCI. However the most widespread BCI systems are based on EEG recordings. BCI consists of a brain signal acquisition system, data processing software for feature extraction and pattern classification, and a system to transfer commands to an external device and, thus, providing feedback to an operator. The most prevalent BCI systems are based on the discrimination of EEG patterns related to execution of different mental tasks [14, 21, 24]. This approach is justified by the presence of correlation between brain signal features and tasks performed, revealed by basic research [24, 28, 30, 45]. By agreement with the BCI operator each mental task is associated with one of the commands to the external device. Then to produce the commands, the operator switches voluntary between corresponding mental tasks. If BCI is dedicated to control device movements then psychologically convenient mental tasks are motor imaginations. For example, when a patient controls by BCI the movement of a wheelchair its movement to the left can be associated with the imagination of the left arm movement and movement to the right - with right arm movement. Another advantage of these mental tasks is that their performance is accompanied by the easily recognizable EEG patterns. Moreover, motor imagination is considered now as an efficient rehabilitation procedure to restore movement after paralysis [4]. Thus, namely the analysis of BCI performance based on motor imagination is the object of the present chapter. 
The most stable electrophysiological phenomenon accompanying motor performance is the decrease of EEG mu-rhythm recording from the central electrodes located over the brain areas representing the involved extremity [29]. This decrease (Event Related Desynchronization, ERD) occurs also when the subject observes the movement of another person [34] and during motor preparation and imagination [30]. In the state of motor relaxation the increase of EEG mu-rhythm is observed [29] which is called Event Related Synchronization (ERS). The exposure of ERD and ERS in specific brain areas during motor imagination of different extremities is the reason of the high efficiency of BCI based on motor imagination [32]. From the other hand, BCI training allows to stabilize and to contrast brain activity corresponding to different mental tasks and hence to facilitate the search of brain areas involved in their performance.

Until now the most widespread technique to localize brain functions is fMRI study which provides high spatial but low temporal resolution. By contrast EEG study provides high temporal but low spatial resolution. The most prospective seems to be the combination of these techniques [9] especially if to take into account the fast progress in methods of solving inverse EEG problem [13,19] dedicated to localize sources of brain activity by distribution of electric potential over head surface. One of the approaches towards the integration of these techniques was suggested in our previous work [11]. Here we develop the approach and apply it to the analysis of more mental states used for BCI control.

\section{Methods}

\subsection{Experimental procedure}

Eight subjects ( 4 male, 4 female) aged from 25 to 65 participated in the study. All subjects were right-handed and had no neurological diseases. The subjects have provided written participation consent. The experimental procedure was approved by the Board of Ethics at the Institute for Higher Nervous Activity and Neurophysiology of the Russian Academy of Sciences.

The experiment with each subject was conducted for 10 experimental days, the one series per day. Each series consisted of training and testing sessions (Fig. 1 A). The first, training, session was designed to train BCI classifier. The following, testing session was designed to provide subjects with the output of the $\mathrm{BCI}$ classifier in real time to enhance their efforts to imagine a movement. The subjects had to perform one of the four instructions presented on a screen of a monitor: to relax and to imagine the movement of the right or left hand or feet. The movement which they were asked to imagine was a handgrip or feet pressure.

Subject was sitting in a comfortable chair, one meter from a 17" monitor, and was instructed to fix a gaze on a motionless circle $(1 \mathrm{~cm}$ in diameter $)$ in the middle of the screen. Four gray markers were placed around the circle to indicate the mental task to be performed. The change of the marker color into green signaled the subject to perform the corresponding mental task. Left and right markers corresponded to left and right hand movement imagining respectively. The lower marker corresponded to feet movement imagining and the upper one corresponded to relaxation. Each command was displayed for 10 seconds. Each clue was preceded by a 4 -second warning when the marker color changed into blue. 
Four such instructions presented in random order constituted a block, one block constituted a training session and nine blocks a testing session (Fig. 1). Thus each subject received 10 blocks of instructions at each experimental day.

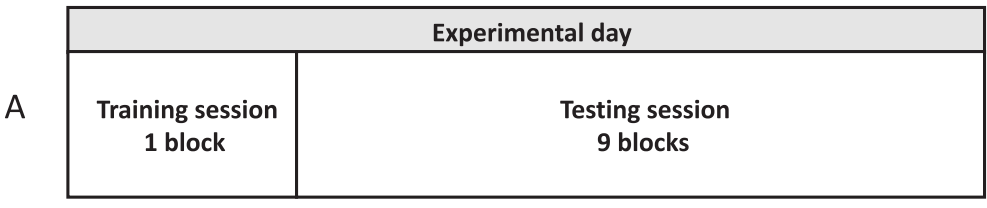

\begin{tabular}{|c|c|c|c|c|c|c|c|}
\hline \multicolumn{8}{|c|}{ Block } \\
\hline \multicolumn{2}{|c|}{ Relaxation } & \multicolumn{2}{|c|}{ Left hand MI } & \multicolumn{2}{|c|}{ Right hand MI } & \multicolumn{2}{|c|}{ Foot MI } \\
\hline 4 & 10 & 4 & 10 & 4 & 10 & 4 & 10 \\
\hline
\end{tabular}

Figure 1. Schematic representation of the experimental protocol and the stages of each experimental session. A. The sequence of sessions. B. The structure of the experimental block. In each block each instruction was presented once. The sequence of their presentation in each block was random. The duration of instructions is given in seconds. Green areas - instructions for performance, blue areas - warnings

During the training session classifier was switched off and recording was used only for its learning. During the following testing sessions classifier was switched on and the result of classification was presented to a subject by color of the central circle. The circle became green if the result coincided with the instruction and its brightness increased with the increase of classifying confidence. During the instruction to relax the presentation of classifying result was switched off not to attract the subject's attention.

EEG was recorded by 48 active electrodes using g.USBamp and g.USBamp API for MATLAB (g-tec, Graz, Austria) with sampling frequency $256 \mathrm{~Hz}$ and filtered by notch filter to suppress supply noise. Electrode positions were Fz, F3, F4, Fcz, Fc3, Fc4, F7, F8, Fcz, Fc3, Fc4, Fc5, Fc6, Fc7, Fc8, Cz, C1, C2, C3, C4, C5, C6, T7, T8, Cpz, Cp1, Cp2, Cp3, Cp4, Cp5, Cp6, Tp7, Tp8, Pz, P1, P2, P3, P4, P5, P6, P7, P8, Poz, Po3, Po4, Po7, Po8, Oz, O1, O2. Afz was a reference.

The data of the last BCI session were used for solving inverse EEG problem, that is to localize the sources of EEG signals inside the brain. We solved it taking into account individual geometry of brain and its covers. To identify the source positions relative to the brain structures, electrodes positions have to be also identified relative to these structure. Since brain structures are given in MRI coordinate system electrode positions have to be also identified in MRI coordinate system. To that end not moving the cap on the subject head we removed electrodes from nodes at the cap and replaced them by small pellets visible on MRI slices (Fig. 2). Just after this procedure the subject was placed in MRI chamber. Since the position of each electrode was marked by the pellet, then identification of the pellet positions on slices allowed to obtain the electrodes coordinates in MRI reference frame.

During fMRI recording instructions to relax or to imagine hand or feet movements were presented to the subject without EEG recording. Since the efficiency of BCI control depends 

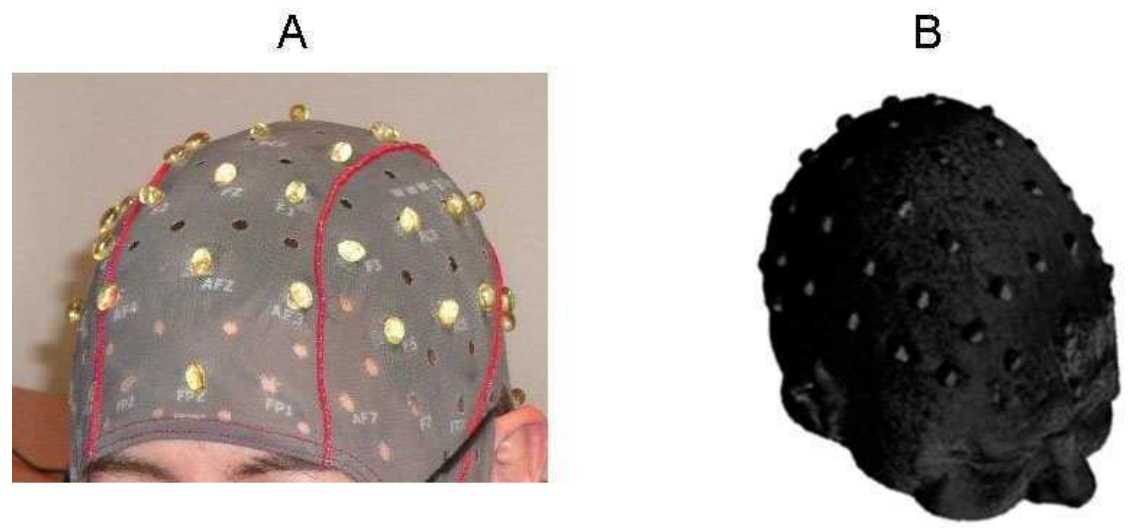

Figure 2. Pellets marking electrode positions on the head surface (A) and their images reconstructed by MRI slices (B).

on the subject's ability to stabilize and to contrast patterns of the brain activity following different instructions, then we believe that the subjects who demonstrate an efficient BCI control are able to produce stable and contrast patterns of the brain activity during both EEG and fMRI experiments. The fMRI examinations were conducted with 3T MR scanner (Siemens Trio Tim, Erlangen, Germany) using 12-channel head coil. Functional MRI data were acquired with a T2*weighted gradient echo EPI sequence ( 40 slices, TR=2500 ms, TE=30 ms, $64 \times 64$ matrix, FOV=192 $\times 192 \mathrm{~mm}$, slice thickness $3 \mathrm{~mm}$, voxel size $=3 \times 3 \times 3 \mathrm{~mm}$, $\mathrm{BW}=2232 \mathrm{~Hz} /$ pixel, axial orientation). Total of 240 dynamic measurements were acquired during fMRI scans resulting to 10 minutes of acquisition time. A T1-weighted anatomical scan using MP-RAGE sequence ( 224 slices, TR=2300 ms, TE $=4.64 \mathrm{~ms}, \mathrm{FOV}=256 \times 256$, slice thickness $1 \mathrm{~mm}$, voxel size of $1 \times 1 \times 1 \mathrm{~mm}, \mathrm{PAT}=2$, sagittal orientation) was also acquired for each subject. The measurement lasted of $5 \mathrm{~min}$.

\subsection{Classifier}

One crucial part of a BCI system is the EEG pattern classifier which can be designed by many methods [1]. We used in our experiments the simplest Bayesian classifier based on EEG covariance matrices. As shown in [10] it provides classification accuracy comparable with other more sophisticated classifiers.

Suppose that there are $L$ different mental tasks to be distinguished and probabilities of each task to be performed are equal to $1 / \mathrm{L}$. Let also for each mental task the EEG signal distribution be Gaussian with zero mean. Also, let $\mathbf{C}_{i}$, a covariance matrix of the signal corresponding to execution of the $i$-th mental task $(i=1, \ldots, L)$, be non-singular. Then probability to obtain EEG signal $\mathbf{X}$ under the condition that the signal corresponds to performing the $i$-th mental task is $P(\mathbf{X} \mid i) \propto e^{-\frac{V_{i}}{2}}$, where $V_{i}=\mathbf{X}^{\mathrm{T}} \mathbf{C}_{i}^{-1} \mathbf{X}+\ln \left(\operatorname{det}\left(\mathbf{C}_{i}\right)\right)$. Following the Bayesian approach, the maximum value of $P(\mathbf{X} \mid i)$ over all $i$ determines the class to which $\mathbf{X}$ belongs. Hence, the signal $\mathbf{X}$ is considered to correspond to execution of 
the $k$-th mental task as soon as $k=\operatorname{argmin}_{i}\left\{V_{i}\right\}$. The equality $\mathbf{X}^{\mathrm{T}} \mathbf{C}_{i}^{-1} \mathbf{X}=\operatorname{trace}\left(\mathbf{C}_{i}^{-1} \mathbf{X} \mathbf{X}^{\mathrm{T}}\right)$ implies that

$$
V_{i}=\operatorname{trace}\left(\mathbf{C}_{i}^{-1} \mathbf{X} \mathbf{X}^{\mathrm{T}}\right)+\ln \left(\operatorname{det}\left(\mathbf{C}_{i}\right)\right)
$$

In practice all $V_{i}$ are rather variable, so it is more beneficial to split signal into epochs and compute average value $\left\langle V_{i}\right\rangle$ for each EEG epoch to be classified, using equation (1)

$$
\left\langle V_{i}\right\rangle=\operatorname{trace}\left(\mathbf{C C}_{i}^{-1}\right)+\ln \left(\operatorname{det}\left(\mathbf{C}_{i}\right)\right)
$$

where $\mathbf{C}$ denotes an epoch data covariance matrix estimated as $\left\langle\mathbf{X} \mathbf{X}^{\mathrm{T}}\right\rangle$. Therefore to perform the classifier training it is sufficient to compute the covariance matrices corresponding to each mental task. It makes $\mathrm{BC}$ computationally inexpensive.

During testing sessions covariance matrix $\mathbf{C}$ was calculated every $250 \mathrm{msec}$ over 1 second sliding window. Respectively, the variable $s$ defining the accuracy of brain state recognition was updated each $250 \mathrm{msec}$. It took 1 if the state recognized corresponded to the instruction and 0 in the opposite case. Then the variable $S$ was updated according to the formula $S=\left(1-\gamma_{s}\right) S+\gamma_{s} s$. At the beginning of new instruction presentation $S$ was set to be zero. The brightness of the central circle which indicated the quality of BCI performance was proportional to $S$. The value of $\gamma_{s}$ was taken to be 0.1 . So the characteristic time of the biofeedback was about $2.5 \mathrm{sec}$.

The covariance matrices $\mathbf{C}_{i}$ were calculated during the training session and then updated during the testing session at the end of each block according to the formula $\mathbf{C}_{i}=(1-$ $\left.\gamma_{c}\right) \mathbf{C}_{i}+\gamma_{c} \mathbf{c}_{i}$ where $\mathbf{c}_{i}$ is a covariance matrix for the $i$-th state calculated over block data. In our experiments we used $\gamma_{c}=0.1$.

The quality of BCI performance was estimated by the results of on-line classifying during the testing session of each experimental day and offline by the data obtained during both training and testing sessions. For offline analysis the data were additionally filtered within 5-30 Hz bandpass. Then 7 blocks of 10 were randomly chosen for classifier learning, i.e. for calculation of covariance matrices for all mental states. Recordings of the remaining 3 blocks were split into epochs of 1 second length. These epochs were used for classifier testing. 50 such classification trials were made. Averaging over all classification trials resulted in $L \times L$ confusion matrix $\mathbf{P}=\left(p_{i j}\right)$. Here $p_{i j}$ is an estimate of probability to recognize the $i$-th mental task in case the instruction is to perform the $j$-th mental task.

We chose the mean probability of correct classification $p$, mutual information $g$ between states recognized and instructions presented, and Cohen's $\kappa$ as indices of classification efficacy. 
Given the confusion matrix $\mathbf{P}$ these indices can be calculated as follows:

$$
\begin{aligned}
& p=\frac{1}{L} \sum_{i=1}^{L} p_{i i}, \\
& g=-\sum_{i, j=1,1}^{L, L} p_{i j} p_{0 j} \log _{2}\left(p_{i j} / p_{i 0}\right) \\
& \kappa=\frac{\sum_{i=1}^{L} p_{i i} p_{0 i}-\sum_{i=1}^{L} p_{0 i} p_{i 0}}{1-\sum_{i=1}^{L} p_{0 i} p_{i 0}}
\end{aligned}
$$

where $p_{0 j}=1 / L$ is probability of the $j$-th instruction to be presented and $p_{i 0}=\sum_{j=1}^{L} p_{i j} p_{0 j}$ is probability of the $i$-th mental state to be recognized.

The better classifier performs the more confusion matrix is close to identity matrix. In case $L$ states are classified perfectly $p=1, g=\log _{2} L$, and $\kappa=1$. If classification is random, i.e. $p_{i j}=p_{i 0}$ for all $j$, then $p=1 / L, g=0$, and $\kappa=0$.

Index $p$ has an advantage of being evidently interpreted as the percentage of correct classification while its disadvantages are, first, that it does not account for distribution of errors since it does not depend on nondiagonal elements of confusion matrix, second, its lower value depends on the number of states classified. For example the value $p=0.5$ corresponds to a random recognition when $L=2$ and twice exceeds the random level when $L=4$. Thus it is difficult to compare the qualities of BCIs with different states classified. Both indices $g$ and $\kappa$ have the advantage of considering the error distribution over nondiagonal elements of confusion matrix and of being normalized to the case of random classifying independently of $L$. In this case both indices are equal to zero. Moreover, $\kappa$ is also normalized to the case of perfect classifying. It takes one in this case, while $g$ depends on $L$. Thus, on one hand $\kappa$ is more convenient for comparing qualities of BCI with different $L$, but on another hand $g$ gives the estimate of BCI quality directly in the rate of information transfer. Since $g$ is calculated every second then it can be measured in bits per second. Thus all three indices are reasonable.

When all probabilities of correct classification are equal, i.e. $p_{i i}=p$ for all $i$, and all probabilities of incorrect classification are equal, i.e. $p_{i j}=(1-p) /(L-1)$ for all $i \neq j$, the mutual information between instructions presented and the states classified can be obtained as:

$$
g=\log _{2} L+p \log _{2} p+(1-p) \log _{2}\left(\frac{1-p}{L-1}\right)
$$

Based on [43], (4) is often used to estimate BCI efficacy ([3], [5] [44]). But if the corresponding assumptions do not hold true, the value of $g$, calculated according to (4), is lower than the actual mutual information. In this study we used the general formula (3). 
To estimate the on-line BCI quality we used confusion matrix obtained over the data of testing session based on EEG signal classification every $250 \mathrm{msec}$ as described above.

\subsection{ICA}

To identify the sources of brain activity the most relevant to BCI performance we used Independent Component Analysis (ICA). Last years ICA becomes widely used in EEG processing, particularly in BCI studies [16]. ICA provides representation of a multidimensional EEG signal $\mathbf{X}(t)$ (where components of $\mathbf{X}(t)$ represent electric potentials recording from $N$ individual electrodes at the head surface) as a superposition of activities of independent components $\xi$ :

$$
\mathbf{X}(t)=\mathbf{W} \xi(t)=\mathbf{W}_{1} \xi_{1}+\mathbf{W}_{2} \xi_{2}+\cdots+\mathbf{W}_{N} \xi_{N}
$$

Columns $\mathbf{W}_{i}$ of matrix $\mathbf{W}$ specify the contribution of the corresponding independent component (or source) into each of the electrodes and the components $\xi_{i}$ of the vector $\xi(t)$ specify sources intensity in each time point. The combination of active sources is supposed to be specific and individual for each mental task. Thus their activities in many tasks can be treated as independent.

There exist a lot of methods to represent the signal $\mathbf{X}$ in the form (5). We used algorithm RUNICA (MATLAB toolbox EEGLab,[7]). RUNICA provides the identification of the independent components maximizing distinction of their distributions from normal one in terms of kurtosis [15]. The using of this method is reasonable because it corresponds to the suggestion that activities of sources are different in different mental states: in some states they expose ERD, in others - ERS. Thus in one state the distribution of EEG amplitude should be narrow, in others - wide. So the common distribution would be maximally different from the normal one.

To reveal the sources of brain activity the most significant for BCI performance the quality index $\kappa$ was calculated in dependence on the number $N_{c m p}$ of ICA components used for mental state classifying. For each $N_{c m p}$ we found the optimal combination of components providing the highest $\kappa$. Since the total number $2^{N}$ (where $N=48$ ) of possible component combinations is extremely large we used exhaustive search to find the optimal combination of components only for $N_{c m p}=3$. To find the optimal combination of components for $N_{c m p}>3$ we used a "greedy" algorithm which added components one by one starting from the optimal combination of 3 components. At each step a component was added to the optimal component combination found at the previous step, so that extended combination provided the highest $\kappa$.

When all ICA components are used for mental states classifying, i.e. $N_{c m p}=N$, then $\xi=$ $\mathbf{W}^{-1} \mathbf{X}$ where $\mathbf{W}^{-1}$ is non-singular. In this case $\mathbf{C}_{i}^{\xi}=\mathbf{W}^{-1} \mathbf{C}_{i}\left(\mathbf{W}^{-1}\right)^{T}$ and

$$
\begin{aligned}
V_{i}^{\xi} & =\operatorname{trace}\left(\left(\mathbf{C}_{i}^{\xi}\right)^{-1} \xi \xi^{T}\right)+\ln \left(\operatorname{det}\left(\mathbf{C}_{i}^{\tau}\right)\right)=\operatorname{trace}\left(\left(\mathbf{C}_{i}\right)^{-1} \mathbf{X} \mathbf{X}^{T}\right) \\
& +\ln \left(\operatorname{det}\left(\mathbf{C}_{i}\right)\right)-2 \ln (\operatorname{det}(\mathbf{W}))=V_{i}-2 \ln (\operatorname{det}(\mathbf{W}))
\end{aligned}
$$


Since $\ln (\operatorname{det}(\mathbf{W}))$ does not depend of the mental state, then $V_{i}^{\zeta}$ and $V_{i}$ reach minima for the same mental state and the mental state classified by Bayesian classifier in terms of $\xi$ coincides with that classified in terms of $\mathbf{X}$. Thus, the case $N_{c m p}=N$ directly corresponds to $\mathrm{BCI}$ performance based on classifying the original signal $X$.

\subsection{Sources localization}

With respect to the EEG analysis, it is reasonable to assume that independent sources of electrical brain activity recorded at the head surface are current dipoles distributed over the neocortex. As shown below our experiments confirm this assumption and at least for the sources the most relevant for BCI performance the distribution of electrical potential over the head produced by each of these sources could be actually interpreted in terms of electrical field produced by single current dipole. Thus, for each of such sources its localization was searched in a single dipole approximation. In other words, position and orientation of a single current dipole were searched which provided maximal matching between patterns of EEG distribution on the head surface given by ICA and by a dipole. The pattern of EEG distribution for dipole with given position and orientation was calculated by solving the direct EEG problem.

It was solved by the finite element method (FEM) which allows to take into account individual geometry of the brain and its covers. In FEM, one critical requirement for the mesh generation is to represent the geometric and electrical properties of the head volume conductors. To generate the FEM meshes from the MRI data, MR images were segmented into five sub-regions: white matter, gray matter, cerebrospinal fluid (CSF), skull and scalp. The segmentation of the different tissues within the head was made by means of SPM8 New Segmentation Tool. To construct the FE models of the whole head the FEM mesh generation was performed using tetrahedral elements with inner-node spacing of $2 \mathrm{~mm}$. Thus, the total number of nodes amounted to about 1.5 millions. Electrical conductivities were assigned to the tissues segmented in accordance with each tissue type: $0.14 \mathrm{~S} / \mathrm{m}$ for white matter, 0.33 $\mathrm{S} / \mathrm{m}$ for gray matter, $1.79 \mathrm{~S} / \mathrm{m}$ for CSF, $0.0132 \mathrm{~S} / \mathrm{m}$ for skull, and $0.35 \mathrm{~S} / \mathrm{m}$ for scalp $[17,46]$. To solve the EEG forward problems, the FEM mesh along with electrical conductivities were imported into the commercial software ANSYS (ANSYS, Inc., PA, USA).

\section{Results}

\subsection{The ICA components most relevant for BCI control}

For all subjects three indices averaged over all experimental days are shown in Fig. 3. The subjects are ranged according to mean $\kappa$ computed for on-line classification. Note that subjects ranking according to $p$ completely preserves their order and according to $g$ changes it only slightly (S5 must be shifted before S3). Thus all indices give good relative estimation of subject ability to control BCI. Mean on-line and offline estimates of BCI performance by all indices are shown to be very close. However on-line estimations are more variable. This is reasonable because on-line estimation is based on one classification trial obtained directly during experiment performance, while offline estimation is based on 50 classification trials as described above. Therefore, confusion matrix computed offline is more confident than that computed on-line. 


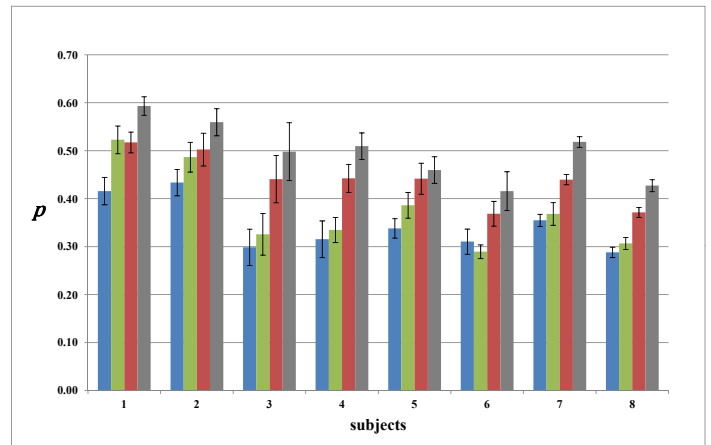

(a)

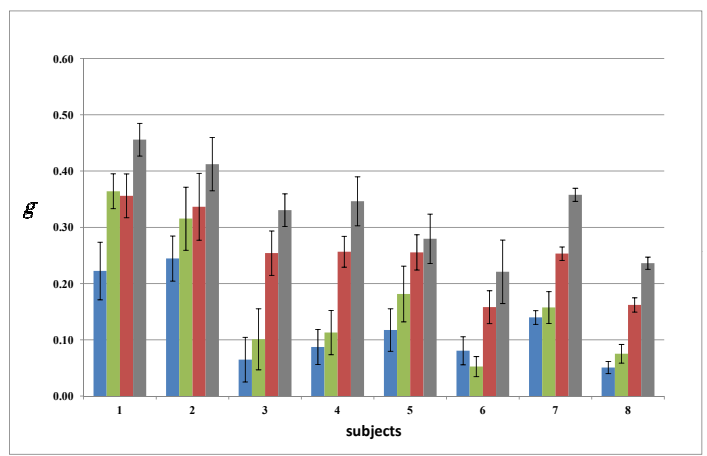

(b)

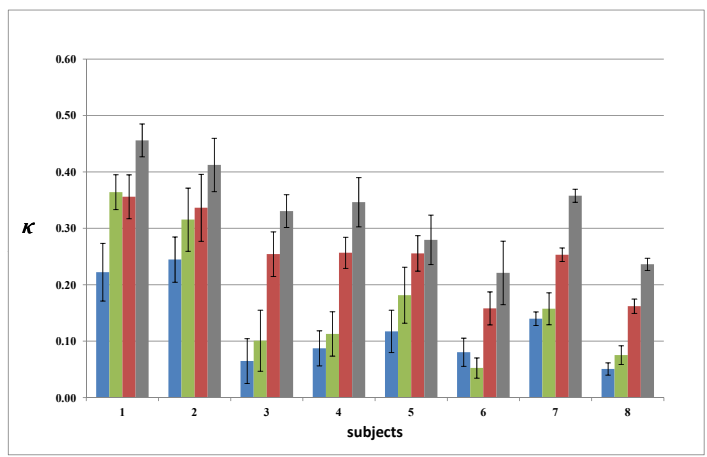

(c)

Figure 3. Indices $p, g$ and $\kappa$ of $\mathrm{BCl}$ control accuracy for all subjects averaged over all experimental days (means and standard errors). Blue bars - on-line, green bars - offline, red - three the most relevant components, grey - optimal components. The subjects are ranged according to $\kappa$ computed on-line. The most relevant components and optimal components were calculated to maximize $\kappa$. Then these components were used to compute $p$ and $g$. 
Fig. 4 demonstrates the dependency of $\kappa$ on the number $N_{c m p}$ of ICA components for all subjects on the last experimental day. For each $N_{c m p}$ index $\kappa$ is shown for individual optimal component combination.

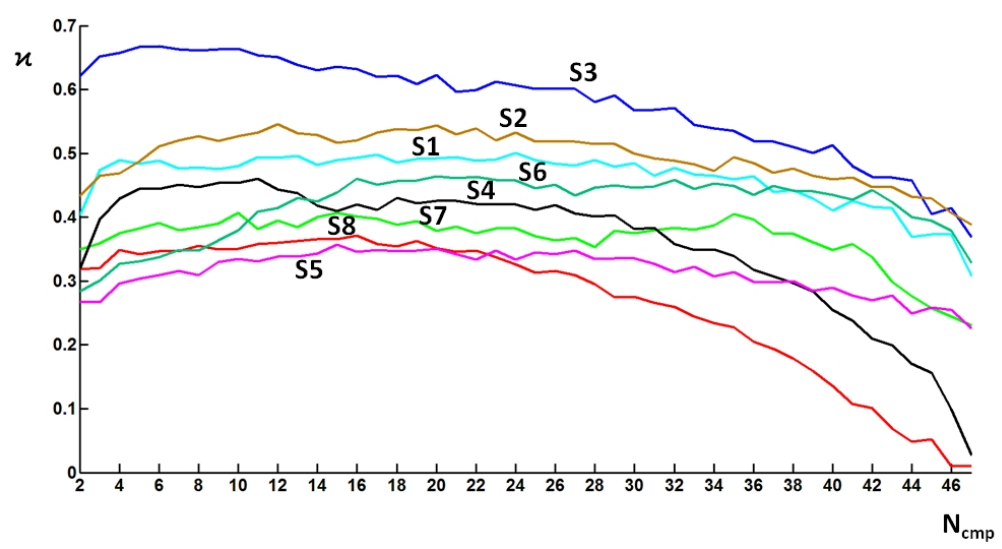

Figure 4. Index $\kappa$ of $\mathrm{BCl}$ control accuracy in dependence on the number $N_{c m p}$ of ICA components used for mental states classifying. The optimal combination of three components $\left(N_{c m p}=3\right)$ was obtained by the exhausted search. The other optimal combinations were obtained by the "greedy" algorithm. Each curve represents the data for each individual subject obtained for the last day of $\mathrm{BCl}$ training.

As shown in Fig. $4 \kappa$ depends on $N_{c m p}$ not monotonically and reaches maximum when some ICA components are discarded. However the classifying accuracy for $N_{c m p}=3$ is very close to that obtained for optimal combination of components providing maximal $\kappa$. Thus the obtained combinations of 3 components can be considered as good representations of the components which are the most relevant for BCI performance. Indices $p, g$ and $\kappa$ for three the most relevant components and for optimal components averaged over all experimental days are shown in Fig. 3 for all subjects. As shown, elimination of not relevant (noisy) components improves BCI performance several times. Basing on the most relevant and optimal components BCI classifier provides the accuracy of mental state recognition significantly exceeding the random level for all subjects and all indices (t-test, $P<0.001$ ). For Subject 1 index $g$ computed for optimal components reached 0.83 that is twice more than $g$ computed on-line for this subject by the original EEG signal. This maximal value can be also compared, for example, with the maximal value of $g=0.58$ obtained for Berlin BCI [5] in experiments with a large group of untrained subjects. Thus, filtering of noisy ICA components is an efficient method to improve BCI performance. Note that although filtering of noisy components essentially improves BCI performance for all subjects it almost preserves the order of their ability to control BCI. Thus the relative subjects skill is mainly determined by their inherent properties but not by the properties of BCI classifier. Note also that the quality of $\mathrm{BCI}$ performance is rather variable over experimental days for each subject. Due to this variability the order of subjects ability to control BCI was not the same every day. For example, as shown in Fig. 4 on the last experimental day the quality of BCI control for S3 was higher than for subjects S1 and S2, although on average it was lower. 
For each experimental day three the most relevant components and optimal components were chosen to maximize $\kappa$ for the data of namely this day. The components happened to be not identical for all experimental days and all subjects but some of them appeared very repeatedly. Four such components which appeared most often among the most relevant ones over all subjects and all experimental days are shown in Fig. 5. The features of these components are shown in terms of their contribution into EEG electrodes (topoplots) and their spectrograms for four considered mental states: relaxation, right or left hand movement imagination and feet movement imagination. The data relates to the last experimental day of Subject 1 who showed the best BCI control on average.

Three of these components $(\mu 1, \mu 2$ and $\mu 3)$ demonstrate very well exposed Event Related Desynchronization (ERD) of mu-rhythm. For the component denoted $\mu 1$ mu-rhythm is suppressed during the left hand motor imagery. Its focus is in the right hemisphere presumably above the primary sensorimotor areas presenting the left hand. Respectively, for the components $\mu 2$ and $\mu 3 \mathrm{mu}$-rhythm is suppressed during the right hand and feet motor imagery and their foci are presumably above the areas presenting right hand and feet. The fourth component denoted $\beta$ we ascribed to the activity of supplementary motor areas (SMA). First, they are located on the midline surface of the hemispheres and hence their activity can produce the focus corresponding to the topoplot of this component. Second, as shown in [27] by electrocorticographic recordings, during the motor act SMA demonstrates ERD in the spectral band $10-40 \mathrm{~Hz}$. Just the same is shown for the component $\beta$. Remind that for our offline analysis EEG signal was filtered in the band $5-30 \mathrm{~Hz}$, so ERD for this component is shown in Fig. 4 up to $30 \mathrm{~Hz}$.

Three of four ICA components discussed here $(\mu 1, \mu 2$ and $\beta)$ are completely identical to those obtained in [11] (Compare Fig. 5 with the first and last rows in Fig. 3 of [11]). Component $\beta$ was identified but not discussed in [11]. Component $\mu 3$ is new because only three mental tasks (relaxation and left or right hand motor imagery but not feet motor imagery) were used for BCI control in [11].

As in [11] components $\mu 1$ and $\mu 2$ demonstrate also well exposed Event Related Synchronization (ERS) during the motor imagery of the opponent extremities. Especially it is seen for $\mu 1$. Mu-rhythm of this component essentially increased during the motor imagery of both right hand and feet. It is worth to note that the topoplots of four described components were very stable for all experimental days but the manifestation of ERD and ERS was rather variable. Namely the variability of their manifestation determined the variability of BCI control quality. However in any case ERD and ERS are much better exposed in terms of ICA components than in direct EEG recording. The level of ERD can be estimated as $r=S_{i m} / S_{r e l}$ where $S_{i m}$ and $S_{r e l}$ are the maximal spectral densities in the alpha band during the motor imagination and relaxation, respectively. For Subject 1 on average over all experimental days and imagination of both hands $r$ amounted to 0.2 in terms of ICA components and to 0.69 for two central electrodes C3 and C4 where ERD was maximally exposed. Thus ICA allowed to rectify ERD and ERS due to excluding the components not exposing these changes of the brain activity.

\subsection{Sources localization}

Although the shown ICA components happened to be rather stable and repeatable over all subjects and all experimental days one could expect that they are only the formal 

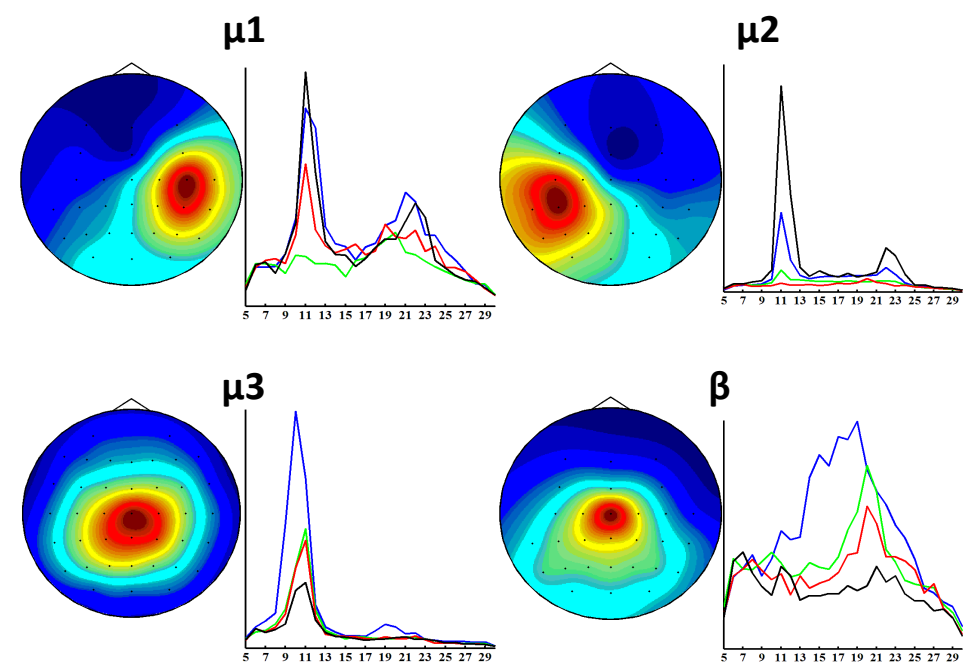

Figure 5. Topoplots and spectrograms for four sources of electrical brain activity the most relevant to $\mathrm{BCl}$ performance. $\mu 1$, $\mu 2$ and $\mu 3$ demonstrate ERD of mu-rhythm during imagination of left hand, right hand and feet movement. $\beta$ demonstrates ERD in the band $10-30 \mathrm{~Hz}$. Spectral frequency is given in $\mathrm{Hz}$, blue curves - relaxation, red lines - right hand motor imagery, green lines - left hand motor imagery, black lines - feet motor imagery.

results of some mathematical transformations of the actual experimental data and have no physiological sense. To clarify their sense we show, first, that their contribution to EEG recordings can be explained by the current dipole sources of brain activity located in the sensorimotor cortical areas, and, second, that locations of these sources coincide with locations of brain activity identified in fMRI study.

As an example, topoplot of $\mu 1$ is compared in Fig. 6 with that produced by a current dipole model found by solving inverse EEG problem. To solve it we found the location and orientation of the dipole provided the best fit between its contribution to EEG electrodes obtained by solving the direct EEG problem and the contribution obtained for $\mu 1$. As shown in Fig. 6 the found dipole provides a good coincidence between both types of contribution. On average over all subject and all components the residual variance of the single dipole approximation amounted only $1 \%$.

Examples of dipole localization along with results of fMRI analysis are shown in Fig. 7. Figure demonstrates voxels for which BOLD level was significantly higher left hand (a), right hand (b), and feet (c) motor imagery compared to relaxation.

Dipole positions obtained for components $\mu 1$ and $\mu 2$ happened to be very close to the sensorimotor "hand areas" marked in Fig. 7a,b. Dipole position obtained for component $\mu 3$ is close to the sensorimotor "feet areas" in the superior regions of post- and precentral gyri (Fig.7c) and for component $\beta$ slightly more anterior, i.e. close to SMA. However, the positions of dipoles corresponding to $\mu 1, \mu 2$ and $\mu 3$ happened to be a little deeper than foci of fMRI activity near "hand" and "feet" areas. Small discrepancy (in the limit of 25 


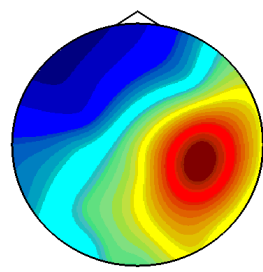

experiment

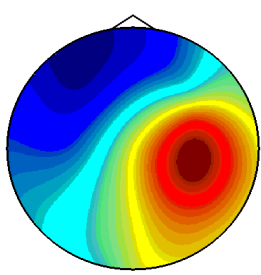

model

Figure 6. Topoplot of component $\mu 1$ (experiment) compared with topoplot produced by a single dipole model (subject S1)
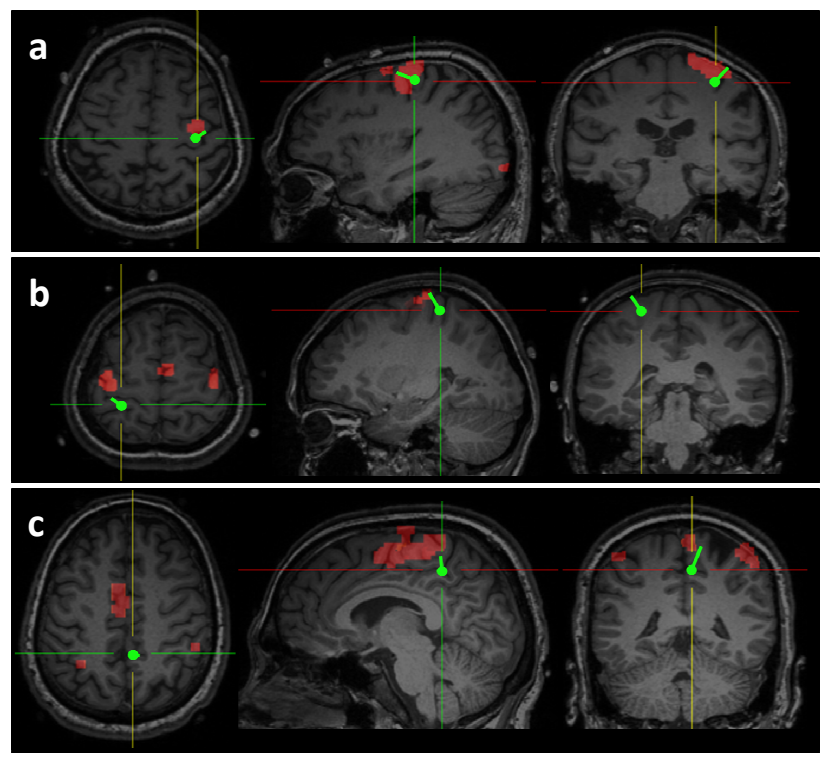

Figure 7. Examples of dipole localization for $\mu 1$ (a), $\mu 2$ (b) and $\mu 3$ (c) components along with the results of fMRI analysis.

$\mathrm{mm}$ ) between current dipole positions and areas of maximal fMRI activity was also obtained in the most studies with measurements of somatosensory activity as a response to hand electrical stimulation (see, for example, $[6,12,41])$. This may be explained by the fact that different processes are responsible for EEG and fMRI outcomes. EEG is the result of neuronal electric activity while fMRI relates to blood flow activity associated with the energetically dominant processes. If, for example, neuronal electric activity in the depth of central sulcus is less energy-expensive than in the crown of pre- and postcentral gyrus than fMRI activity removes up comparing with EEG activity. Moreover, in our experiments a motor imagination results in the increase of fMRI activity but decrease of EEG activity. Since relation between fMRI activity and neuronal electric activity is rather complex [25], then the brain areas where these two kinds of brain activity are maximally exposed could be slightly different. Hence the small discrepancy of their positions does not evidence against the precision of dipole 
location. In our experiments the distance between dipole location and the COM of fMRI activity near central sulcus averaged over subjects and components amounted to $9 \pm 1.5 \mathrm{~mm}$. The dipoles corresponding to components $\mu 1, \mu 2$ and $\mu 3$ were located at the bottom of the central sulcus (Fig. 7), i.e. at the area 3a responsible for proprioceptive sensation. According to reports of the subjects this corresponds to their internal feeling of the imagined movement.

\section{Discussion}

Generally, the difficulties in interpreting the original EEG signals are due to the overlapping of activities coming from different brain sources, due to the distortion of the current flows caused by the inhomogeneity in the conductivity of the brain and its covers and due to uncertainty not only in dipole source locations but also in the dipole orientation which determines the relation between its position and the EEG amplitude maxima which it produces at the head surface. These difficulties result in common notion that EEG data provide high temporal but very low spatial resolution comparing to fMRI data. Last years there were many efforts to match these techniques to enhance both resolutions [25]. One of the approaches is presented here in the chapter. It contains the following steps:

1. Subjects training in BCI control to stabilize and to contrast the patterns of EEG activities related to the mental tasks under consideration.

2. When subjects are sufficiently trained, finding the ICA components which are the most relevant for the $\mathrm{BCI}$ control. This allows to suppress the influence of not relevant sources of brain activity and to refine the most relevant ones.

3. Obtaining of individual geometries of the brain and its covers (e.g. by MRI data) - as result inverse problem can be solved taking geometries into account

4. The inverse EEG problem solving for each individual relevant component. This allows to perform the solution in the single dipole approximation.

5. Verifying the found dipole locations by fMRI data to be sure that the found ICA components are not only the formal results of some mathematical transformations.

This approach allowed us to find the location of the sources of the brain activity which are the most relevant for motor imagination. Three of them denoted as $\mu 1, \mu 2$ and $\mu 3$ happened to be localized at the bottom of central sulcus close to the Brodmann area 3a responsible for proprioceptive sensation. This location corresponds to the internal feeling of the imagined hand or feet movement according to the reports of subjects. Thus, the experience of imagery (at least for motor imagery) involves perceptual structures despite the absence of perceptual stimulation.

There is a long story of the debates concerning the brain areas involved into the motor imagination, especially the involvement of the primary sensorimotor cortex. Activated areas in or around primary motor area have been described in PET studies [40] during imagination of arm movement, but not of the grasping movement [8]. Primary sensorimotor cortex fMRI activation during the motor imagination was denied in $[35,37]$, but was claimed in $[9,20,36]$.

There were also many efforts to reveal whether SM1 is active during motor imagery basing on EEG data $[2,26,30]$. Particularly, in $[26,30]$ the conclusion that it activates was based on the observation that ERD is maximally exposed at the electrodes related to SM1 activity. 
However as mentioned above it is difficult to prescribe electrical activity to some particular brain area on the base of original EEG data. We believe that our approach allows to do this more substantiated. The reasonable and well interpreted results were obtained here due to solving the inverse EEG problem with the data refined by ICA. The components which are the most relevant to the performance of $\mathrm{BCI}$ based on the hand motor imagination were also obtained in [22]. They are very close or may be even identical to the components obtained in the present paper. But in [22] they were not interpreted in terms of dipole sources and consequently the inverse EEG problem was not solved. We tried to solve it with the most realistic head model as a volume conductor taking into account the individual geometry of brain and its covers and the difference in conductivity of white and grey matters, CSF, skull and scalp. The only thing that we ignored is the anisotropy of the white matter (WM). Although as shown in [19] the head model incorporating realistic anisotropic WM conductivity distributions do not substantially improve the accuracy of EEG dipole localization, our next step is to take into account also the anisotropy.

Besides the foci of fMRI activity which were associated with three sources of EEG activity the most relevant for BCI performance (foci in primary sensorimotor areas 3 and 4 shown in Fig. 7) we observed many other foci. Among them are foci in cerebellum, superior temporal area 22, ventral anterior cingulate area 24 and insula. Thus motor imagery involves rather wide brain networks. According to the literature it can involve also superior and inferior parietal lobule, pre-frontal areas, inferior frontal gyrus, secondary somatosensory area and basal ganglia (see, for example $[9,39])$. We also obtained many other ICA components which were relevant to motor imagination except four main components $\mu 1, \mu 2, \mu 3$ and $\beta$. Since we obtained good relation between these components and fMRI data the natural goal of our future research is to reveal the relations between other fMRI foci and other ICA components.

\section{Acknowledgments}

This research has been partly funded by project GACR P202/10/0262, by the IT4Innovations Centre of Excellence project, reg. no. CZ.1.05/1.1.00/02.0070 supported by Operational Programme "Research and Development for Innovations" funded by Structural Funds of the European Union and state budget of the Czech Republic and by long-term strategic development financing of the Institute of Computer Science (RVO:67985807).

\section{Author details}

Alexander Frolov $1,2, \star$, Dušan Húsek ${ }^{3}$, Pavel Bobrov ${ }^{1,2}$, Olesya Mokienko ${ }^{1}$ and Jaroslav Tintera ${ }^{4}$

* Address all correspondence to: aafrolov@mail.ru

1 Institute of Higher Nervous Activity and Neurophysiology, RAS, Moscow, Russia

2 Faculty of Electronics and Informatics, VŠB-Technical University of Ostrava, Ostrava Poruba, Czech Republic

3 Institute of Computer Science, Academy of Sciences of the Czech Republic, Prague, Czech Republic

4 Institute for Clinical and Experimental Medicine, Prague, Czech Republic 


\section{References}

[1] Bashashati A, Fatourechi M, Ward R, Birch G (2007) A survey of signal processing algorithms in brain-computer interfaces based on electrical brain signals. Journal of Neural engineering 4: R32.

[2] Beisteiner R, Hollinger P, Lindinger G, Lang W, Berthoz A (1995) Mental representations of movements. Brain potentials associated with imagination of hand movements. Electroecephalogr. Clin. Neurophysiol. 96:183-193.

[3] Besserve M, Jerbi K, Laurent F, Baillet S, Martinerie J, et al. (2007) Classification methods for ongoing EEG and MEG signals. Biological research 40: 415-437.

[4] Birbaumer N., Cohen L.G. (2007). Brain-computer interfaces: communication and restoration of movement in paralysis, J Physiol., 579, pp. 621-636.

[5] Blankertz B., Dornhege G., Krauledat M., Muller K.R, Curio G. The non-invasive Berlin Brain Computer Interface: Fast acquisition of effective performance in untrained subjects. Neuroimage. 2007. 37(2): 539Ü550.

[6] Christmann C, Ruf M, Braus D F, Flor H (2002) Simultaneous electroencephalography and functional magnetic resonance imaging of primary and secondary somatosensory cortex in humans after electric stimulation. Neuroscience Letters 333: 69-73.

[7] Delorme A., Makeig S., 2004, EEGLAB: an open source toolbox for analysis of single-trial EEG dynamics. Journal of Neuroscience Methods 134:9-21

[8] Decety J, Perani D, Jeannerod M, Bettinardi V, Tadary B, Woods R, Mazziotta JC, Fazio F (1994) Mapping motor representations with positron emission tomography. Nature 371: 600-602

[9] Formaggio E, Storti SF, Cerini R, Fiaschi A, Manganotti P (2010) Brain oscillatory activity during motor imagery in EEG-fMRI coregistration. Magnetic Resonance Imaging 28(10):1403-12

[10] Frolov A, Husek D., Bobrov P. (2011) Comparison of four classification methods for brain computer interface. Neural Network World, 21(2) 101-115

[11] Frolov A, Husek D, Bobrov P, Korshakov A, Chernikova L, Konovalov R, Mokienko O (2012) Sources of EEG activity most relevant to performance of brain-computer interface based on motor imagery. NNW 1/12: 21-37.

[12] Del Gratta C, Della Penna S, Ferretti A, Franciotti R, Pizzella V, Tartaro A, Torquati K, Bonomo L, Romani G L, Rossini PM (2002). Topographic organization of the human primary and secondary somatosensory cortices: comparison of fMRI and MEG findings. Neuroimage, 17(3):1373-83.

[13] Grech R, Cassar T, Muscat J, Camilleri KP, Fabri SG, Zervakis M, Xanthopoulos P, Sakkalis V, Vanrumste B (2008) Review on solving the inverse problem in EEG source analysis. Journal of NeuroEngineering and Rehabilitation 5(25): 1-33. 
[14] Haynes J, Rees G (2006) Decoding mental states from brain activity in humans. Nature Reviews Neuroscience 7: 523-534.

[15] Hyvarinen A., Karhunen J., Oje E. (2001) Independent component analysis. Willey, New-York. 480 pp.

[16] Kachenoura A., Albera L., Senhadji L., Comon P. (2008) ICA: a potential tool for BCI systems. IEEE Signal Processing Magazine 25(1) 57-68

[17] Kim TS, Zhou Y, Kim S, Singh M (2002). EEG distributed source imaging with a realistic finite-element head model. IEEE Trans Nucl Sci; 49:745-52.

[18] Kohavi R, Provost F (1998): Glossary of terms. Machine Learning - Special Issue on Applications of Machine Learning and the Knowledge Discovery Process. 30, 271-274.

[19] Lee WH, Liu Z, Mueller BA, Limb K, He B (2009). Influence of white matter anisotropic conductivity on EEG source localization: Comparison to fMRI in human primary visual cortex. Clin Neurophysiol 120(12): 2071-2081

[20] Leonardo M, Fieldman J, Sadato N, Campbell G, Ibanez V, Cohen L, Deiber M-P Jezzard P, Pons T, Turner R, Le Bihan D, Hallett M (1995) A functional magnetic resonance imaging study of cortical regions associated with motor task execution and motor ideation in humans. Hum. Brain. Mapp. 3: 135-141.

[21] Leuthardt E, Schalk G, Roland J, Rouse A, Moran D (2009) Evolution of brain-computer interfaces: going beyond classic motor physiology. Neurosurgical focus 27: E4.

[22] Lou B., Hong B., Task-irrelevant alpha component analysis in motor imagery based brain computer interface. 30th Annual International IEEE EMBS Conference, Vancuver, Canada, 2008, 1021-1024.

[23] Mellinger J, Schalk G, Braun C, Preissl H, Rosenstiel W, Birbaumer N, Kubler A (2007) An MEG-based brain-computer interface (BCI). Neuroimage. 36: 581-593.

[24] Millan J, Mourino J, Marciani M, Babiloni F, Topani F, et al. Adaptive brain interfaces for physically-disabled people; 1998. Citeseer. pp. 2008-2011.

[25] Mulert C, Lemieux L (Eds) (2010) EEG-fMRI. Physiological basis, techniques and application. Springer

[26] Neuper C, Scherer R, Reiner M, Pfurtscheller G (2005) Imagery of motor actions: Differential effects of kinesthetic and visual-motor mode of imagery in single-trial EEG. Cognitive Brain Reserch 25: 668-677.

[27] Ohara S, Ikeda A, Kunieda T, Yazawa S, Baba K, Nagamine T, Taki W, Hashimoto N, Mihara T, Shibasaki H (2000) Movement-related changes of electrocorticographic activity in human supplementary motor are proper. Brain 123: 1203-1215. 
[28] Pfurtscheller G, Flotzinger D, Kalcher J (1993) Brain-computer Interface-a new communication device for handicapped persons. Journal of Microcomputer Applications 16: 293-299.

[29] Pfurtscheller, G., Neuper, C. (1994). Event-related synchronization of mu rhythm in the EEG over the cortical hand area in man. Neurosci. Lett. 174, 93-96.

[30] Pfurtscheller G, Neuper C, Flotzinger D, Pregenzer M (1997) EEG-based discrimination between imagination of right and left hand movement. Electroencephalography and clinical Neurophysiology 103: 642-651.

[31] Pfurtscheller G, Neuper C (1997) Motor imagery activates primary sensorimotor area in humans. Neuroscience Letters. 239: 65-68.

[32] Pfurtscheller G., Neuper C. (2001) Motor imagery and direct brain-computer communication. Proceedings of the IEEE, 82 (7), 1123-1134.

[33] Pfurtscheller G., Brunner C., Schlogl A., Lopes da Silva F. (2006) Mu rhythm (de) synchronization and EEG single-trial classification of different motor imagery tasks. NeuroImage 31: 153-159.

[34] Pineda J.A. (2005) The functional significance of mu rhythm: Translating "seeing" and "hearing" into "doing". Brain Research Reviews 50: 57- 68.

[35] Rao SM, Binder JR, Bandettini PA, Hammeke TA, Yetkin FZ, Jesmanowicz A, Lisk LM, Morris GL, Mueller WM, Estkowski LD, Wong EC, Haughton VM, Hyde JS (1993) Functional magnetic resonance imaging of complex human movements. Neurology 43: 2311-2318.

[36] Sabbah P, Simond G, Levrier O, Habib M, Trabaud V, Murayama N, Mazoyer BM, Briant JF, Raybaud C, Salamon G (1995) Functional magnetic resonance imaging at $1.5 \mathrm{~T}$ during sensory motor and cognitive tasks. Eur. Neurol. 35: 131-136/

[37] Sanes JN, Stern CE, Baker JR, Kwong KK, Donoghue JP, Rosen BR (1993) Human frontal motor cortical areas related to motor performance and mental imagery. Soc. Neurosci. Abstr. 18: 1208

[38] Sitaram R, Zhang H, Guan C, Thulasidas M, Hoshi Y, Ishikawa A, Shimizu K, Birbaumer N (2007) Temporal classification of multichannel near-infrared spectroscopy signals of motor imagery for developing a brain-computer interface. NeuroImage 34.4, 1416-1427

[39] Solodkin A, Hlustik P, Chen EE, Small SL (2004) Fine modulation in network activation during motor execution and motor imagery. Cerebral Cortex 14.11, 1246-1255

[40] Stephan K M., Fink GR, Passingham RE., Silbersweig D., Ceballos-Baumann AO., Frith CD., Frackowiak RSJ (1995) Functional anatomy of the mental representation of upper extremity movements in healthy subjects. Journal of Neurophysiology, 73 (1): 373-386 
[41] Thees S, Blabkenburg F, Taskin B, Curio G, Villringer A (2003) Dipole source localization and fMRI of simultaneously recorded data applied to somatosensory categorization. NeuroImage 18: 707-719

[42] Weiskopf N, Veit R, Erb M, et al. Mathiak K, Grodd W, Goebel R, Birbaumer N. (2003) Physiological self-regulation of regional brain activity using real-time functional magnetic resonance imaging (fMRI): methodology and exemplary data. Neuroimage. 19: $577-586$

[43] Wolpaw J, Birbaumer N, Heetderks W, McFarland D, Peckham P, et al. (2000) Brain-computer interface technology: a review of the first international meeting. IEEE Transactions on Rehabilitation Engineering 8: 164-173.

[44] Wolpaw J, Birbaumer N, McFarland D, Pfurtscheller G, Vaughan T (2002) Brain-computer interfaces for communication and control. Clinical neurophysiology 113: 767-791.

[45] Wolpaw J., McFarland D.: Control of a two-dimensional movement signal by a noninvasive brain-computer interface in humans. Proceedings of the National Academy of Sciences of the United States of America 101: 17849, 2004

[46] Wolters CH, Anwander A, Tricoche X, Weinstein D, Koch MA, MacLeod RS. Influence of tissue conductivity anisotropy on EEG/MEG field and return current computation in a realistic head model: a simulation and visualization study using high-resolution finite element modeling. NeuroImage 2006;30:813-26. 
